\title{
Gel immersion endoscopic submucosal dissection using a novel gel product for a duodenal epithelial tumor
}

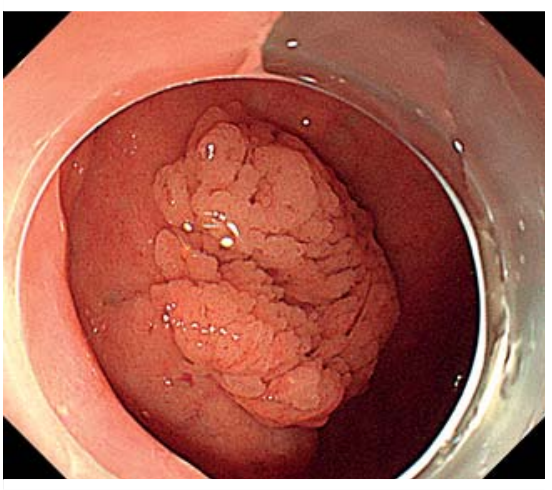

- Fig. 1 Endoscopic image showing a 25-mm flat elevated tumor located in the anterior wall of the duodenal bulb.

Endoscopic submucosal dissection (ESD) for duodenal epithelial tumors is technically difficult, with high risks of perforation and bleeding [1]. Therefore, the development of a safe and reliable endoscopic resection method is warranted for these tumors.

Recently, saline or gel (OS-1 Jelly; Otsuka Pharmaceuticals Factory, Tokushima, Japan) immersion ESD has been performed for a number of gastrointestinal tumors, offering good intraoperative visualization and safe outcomes [2-5]. However, in these reports [2-4], the use of bipolar devices for ESD is recommended, because electrical energy using a monopolar device is dispersed in an electrolyte solution such as saline or OS-1 Jelly, which has an electrical conductivity higher than that of human body tissue. Bipolar devices are not in widespread use; therefore, many ESD operators use a monopolar device and may be unfamiliar with the use of bipolar devices. Herein, we report a technically difficult duodenal ESD using a monopolar device that was successfully performed using a recently developed novel gel with electrolyte removal (Viscoclear; Otsuka Pharmaceuticals Factory). A 57-year-old man was referred to our institute for a flat elevated tumor $(25 \mathrm{~mm}$ in diameter) in the duodenal bulb ( $\bullet$ Fig. 1 ).

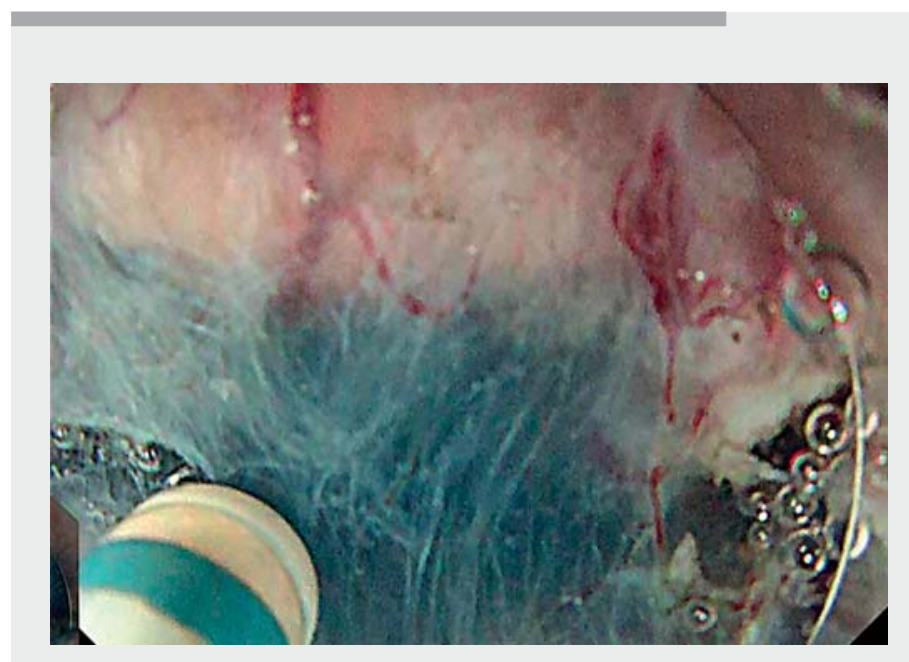

$\checkmark$ Video 1 Successful gel immersion duodenal endoscopic submucosal dissection using a novel gel product for a duodenal epithelial tumor.

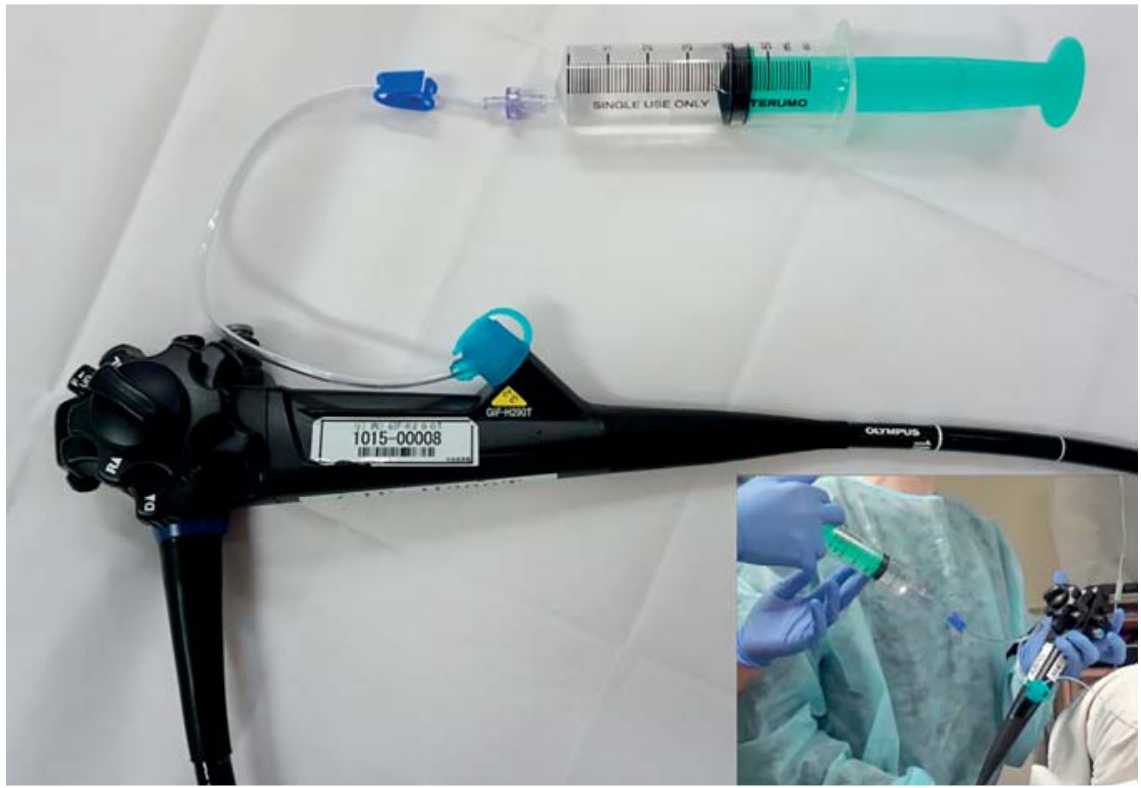

Fig. 2 Photograph of a therapeutic endoscope (GIF-H290T; Olympus, Medical Systems Co., Tokyo, Japan) with the BioShield irrigator (US Endoscopy, Mentor, Ohio) that was used to inject the gel, allowing use of an electrosurgical monopolar knife (DualKnife J; KD655Q Olympus) at the same time through this accessory channel.

During gel immersion ESD ( $\bullet$ Video 1 ), after submucosal injection of hyaluronic acid, we injected gel through the acces- sory channel ( $\triangleright$ Fig. 2 ) without $\mathrm{CO}_{2}$ insufflation. We performed slight submucosal dissection on the proximal side of the tu- 


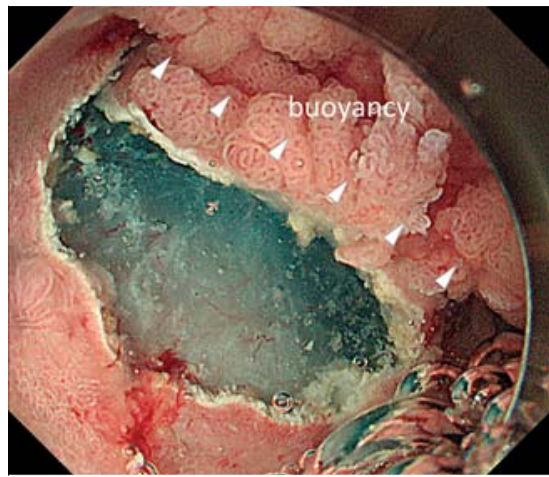

- Fig.3 Gel immersion view showing the buoyancy effect on the tumor (white arrows) after initial mucosal incision and slight submucosal dissection.

mor. As a beneficial effect of solution immersion, the buoyancy effect on the tumor allowed easy insertion of the endoscope under the submucosal layer ( Fig.3). When unexpected bleeding occurred, the gel immersion secured a space for sufficient endoscopic visualization and hemostasis was achieved immediately and easily ( $\triangleright$ Fig.4). The tumor was completely excised in 40 minutes without perforation ( $\triangleright$ Fig. 5).

Gel immersion provided space for endoscopic visualization and submucosal dissection in the duodenum. To the best of our knowledge, this is the first report describing duodenal ESD using this novel gel product.

Endoscopy_UCTN_Code_CCL_1AB_2AZ_3AB

\section{Competing interests}

The authors declare that they have no conflict of interest.

The authors

Tomoaki Tashima' ${ }^{\circledR}$, Kazuya Miyaguchi' ${ }^{1}$, Rie Terada', Yumi Mashimo', Yuki Tanisaka' ${ }^{\complement}$ Tomonori Kawasaki², Shomei Ryozawa'

1 Department of Gastroenterology, Saitama Medical University International Medical Center, Saitama, Japan

2 Department of Pathology, Saitama Medical University International Medical Center, Saitama, Japan

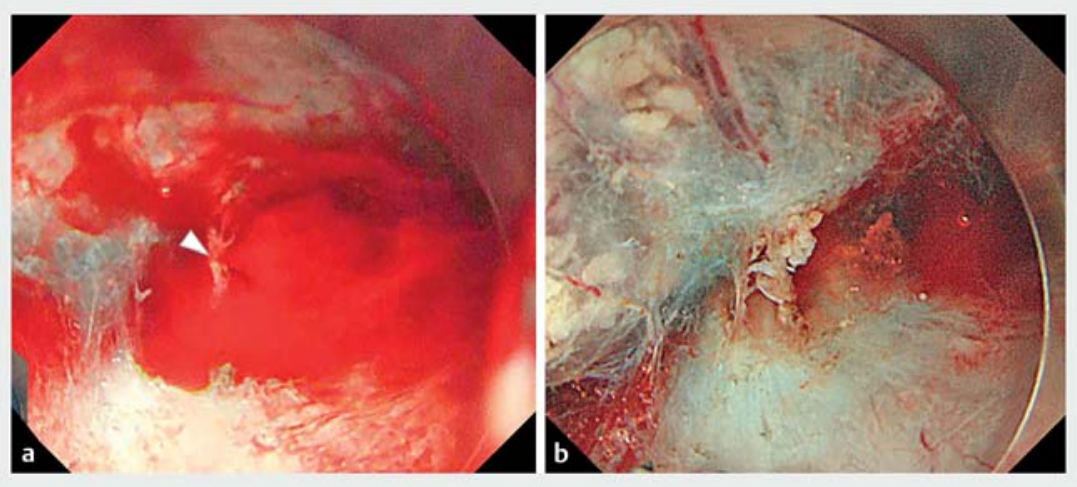

- Fig. 4 Endoscopic images showing: a intraoperative arterial bleeding, with the bleeding point accurately identified (white arrows) after continuous injection of gel; b complete control of bleeding using hemostatic forceps (Coagrasper; Olympus).
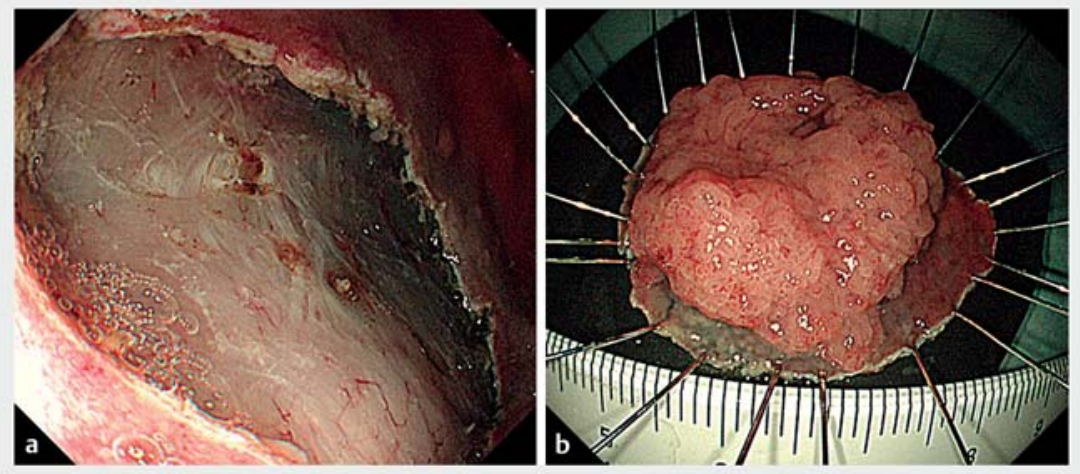

- Fig. 5 The final appearance of: a the mucosal defect with no evidence of perforation; b the resected specimen $(32 \times 26 \mathrm{~mm})$, which was shown on pathology to be a non-ampullary pyloric gland adenoma with negative margins.

\section{Corresponding author}

\section{Tomoaki Tashima, MD, PhD}

Department of Gastroenterology, Saitama Medical University International Medical Center, 1397-1 Yamane, Hidaka City,

Saitama 350-1298, Japan

t.tashima1981@gmail.com

\section{References}

[1] Yahagi N, Kato M, Ochiai Y et al. Outcomes of endoscopic resection for superficial duodenal epithelial neoplasia. Gastrointest Endosc 2018; 88: 676-682

[2] Yoshii S, Akasaka T, Hayashi Y et al. "Underwater" endoscopic submucosal dissection: a novel method for resection in saline with a bipolar needle knife for colorectal epithelial neoplasia. Surg Endosc 2018; 32: 5031 5036
[3] Nagata M. Underwater endoscopic submucosal dissection in saline solution using a bent-type knife for duodenal tumor. VideoGIE 2018; 3: 375-377

[4] Akasaka T, Takeuchi Y, Ishida $\mathrm{H}$ et al. A novel gel immersion technique using a bipolar needle-knife in endoscopic submucosal dissection for superficial gastrointestinal neoplasms. Ann Gastroenterol 2018; 31: 247

[5] Miura Y, Yano T, Takezawa T et al. Gel immersion endoscopy simplifies hemostasis during endoscopic submucosal dissection using the pocket-creation method. Endoscopy 2018; 50: E294-E295

\section{Bibliography}

Endoscopy 2022; 54: E162-E163

DOI $10.1055 / a-1443-4796$

ISSN 0013-726X

published online 28.4.2021

(c) 2021. Thieme. All rights reserved.

Georg Thieme Verlag KG, Rüdigerstraße 14

70469 Stuttgart, Germany 